\title{
Erratum to: Almost complex structures in 6D with non-degenerate Nijenhuis tensors and large symmetry groups
}

\author{
B. Kruglikov ${ }^{1} \cdot$ H. Winther ${ }^{1}$
}

Published online: 23 February 2017

C) Springer Science+Business Media Dordrecht 2017

\section{Erratum to: Ann Glob Anal Geom (2016) 50:297-314 DOI 10.1007/s10455-016-9513-5}

\begin{abstract}
We correct an error in the second part of Theorem 3 of our original paper.
It was stated in [2, Theorem 3] that non-degenerate almost complex manifolds $\left(M^{6}, J\right)$ with a symmetry algebra of dimension 9 are all (locally) homogeneous spaces $M=G / H$ and that they have a semi-simple stabilizer $H$. While the first part is correct, the second claim fails as the following shows.
\end{abstract}

Example Let $\mathfrak{a}=\mathbb{R} b \oplus \mathbb{R} z \oplus V^{4}$ be the solvable Lie algebra with $\mathbb{R} z \oplus V^{4}$ a 5D Heisenberg sub-algebra and $b$ acting as a derivation on $\mathbb{R} z \oplus V^{4}$ with weight 1 on $V^{4}$ and 2 on $\mathbb{R} z$. Note that $\mathfrak{a}$ is isomorphic to the radical of the algebra A.3.1 from [1] (for a particular choice of parameters). The outer derivations of $\mathfrak{a}$ are given by

$$
\operatorname{out}(\mathfrak{a}):=\operatorname{der}(\mathfrak{a}) / \mathfrak{a}=\mathfrak{s p}(4, \mathbb{R}),
$$

and the action of $\mathfrak{s p}(4, \mathbb{R})$ is standard on $V^{4}$ and trivial on $\mathbb{R} z \oplus \mathbb{R} b$. There are several inequivalent embeddings of $\mathfrak{s l}(2, \mathbb{R})$ into $\mathfrak{s p}(4, \mathbb{R})$. The two most interesting to us are the embeddings $\mathfrak{s u}(1,1)$, which preserves a complex structure on $V^{4}$, and $\mathfrak{s}_{2}^{\text {irr }}$, which acts irreducibly on $V^{4}$. If we let $\mathfrak{g}=\mathfrak{s u}(1,1) \ltimes \mathfrak{a}$, then $\mathfrak{g}$ is the algebra A.3.1 from [1]. However, we let $\mathfrak{g}=\mathfrak{s l}_{2}^{\text {irr }} \ltimes \mathfrak{a}$ instead. Then $\mathfrak{g}$ is no longer isomorphic to A.3.1, and $\mathfrak{g} / \mathfrak{s} l_{2}^{\text {irr }}$ does not admit an $\mathfrak{s l}_{2}^{\text {irr }}$-invariant almost complex structure, but there is a 3D solvable sub-algebra

The online version of the original article can be found under doi:10.1007/s10455-016-9513-5.

B. Kruglikov

boris.kruglikov@uit.no

H. Winther

henrik.winther@uit.no

1 Department of Mathematics and Statistics, Faculty of Science and Technology, UiT The Arctic University of Norway, 90-37 Troms $\varnothing$, Norway 
$\mathfrak{h}=\mathfrak{l}_{1} \subset \mathfrak{g}$, which has a one-dimensional intersection with $V^{4} \subset \mathfrak{a}$, such that

$$
\mathfrak{m}=\mathfrak{g} / \mathfrak{h}
$$

admits an $\mathfrak{h}$-invariant almost complex structure, and this structure is non-degenerate. There is a basis $z, b, x_{1}, \ldots, x_{4}, h, e, f$ of $\mathfrak{g}$ such that the structure equations of $\mathfrak{g}$ are:

$$
\begin{aligned}
& {\left[b, x_{i}\right]=x_{i}, \quad[b, z]=2 z, \quad\left[h, x_{1}\right]=-3 x_{1}, \quad\left[h, x_{2}\right]=-x_{2}, \quad\left[h, x_{3}\right]=x_{3},} \\
& {\left[h, x_{4}\right]=3 x_{4}, \quad\left[f, x_{2}\right]=-3 x_{1}, \quad\left[f, x_{3}\right]=-2 x_{2}, \quad\left[f, x_{4}\right]=-x_{3}, \quad\left[e, x_{1}\right]=x_{2},} \\
& {\left[e, x_{2}\right]=2 x_{3}, \quad\left[e, x_{3}\right]=3 x_{4}, \quad\left[x_{1}, x_{4}\right]=z, \quad\left[x_{2}, x_{3}\right]=-3 z, \quad[h, f]=-2 f,} \\
& {[h, e]=2 e, \quad[f, e]=h .}
\end{aligned}
$$

In this basis, we have $\mathfrak{l}_{1}=\left\langle x_{1}, h-b, f-z\right\rangle$. Identifying $\mathfrak{m}=\mathfrak{g} / \mathfrak{h}=\left\langle z, b, e, x_{2}, x_{3}, x_{4}\right\rangle$ the almost complex structure is given by $J z=x_{2}, J b=-x_{3}, J e=x_{4}$ (the invariant structure $J$ is unique: the conjugation $J \mapsto-J$ is obtained by the outer automorphism $x_{i} \mapsto-x_{i}$ ).

We also note that there exists a unique (up to scale) invariant almost pseudo-Hermitian metric $(g, J)$ on $\mathfrak{m}$. In the dual basis $z^{*}, b^{*}, x_{1}^{*}, \ldots, x_{4}^{*}: g=e^{*} z^{*}+x_{2}^{*} x_{4}^{*}-b^{* 2}-x_{3}^{* 2}$.

This counter-example was found upon revisiting the result via a new technique. In [2] we relied on a Maple computation with polynomial ideals that express the Jacobi identity for reconstructed Lie algebra structure of the symmetry algebra $\mathfrak{g}$. Some solutions have been lost with this approach. In [3] we elaborated a different reconstruction technique, which effectively separates linear constraints from genuine quadratic relations, and exploring it we obtained the missing case(s), thus complementing the classification of homogeneous spaces (with semi-simple isotropy) performed in [1].

Recall from Section 3 of [2] that the isotropy algebra $\mathfrak{h}$ can be one of the types: $\mathfrak{p}, \mathfrak{r}, \mathfrak{l}_{0}, \mathfrak{l}_{1}$ or $\mathfrak{l}_{2}$. In the cases $\mathfrak{h}$ is $\mathfrak{p}, \mathfrak{l}_{2}$ or $\mathfrak{r}$ (when $s \notin \mathfrak{r}$ ) we computed $H^{1}(\mathfrak{h}, \operatorname{Hom}(\mathfrak{m}, \mathfrak{h}))=0$, so the homogeneous space is reductive and the further computations hold. We should only revisit the remaining cases.

Theorem $3^{+}$Let $(G / H, J)$ be a homogeneous almost complex $6 D$ manifold with nondegenerate Nijenhuis tensor $N_{J}$. If the stabilizer $H$ is not semi-simple, then its Lie algebra is $\mathfrak{h}=\mathfrak{l}_{1}$, and $\mathfrak{g}=\mathfrak{s l}_{2} \ltimes \mathfrak{a}$, with $\mathfrak{s l}_{2}$ acting irreducibly on $V^{4} \subset \mathfrak{a}$ as described in the example above, and $\mathfrak{l}_{1}$ is conjugate to the one given in the example.

Before proving this theorem, we recall the main result of [3]. For $\varphi \in \mathfrak{h}^{*} \otimes \mathfrak{m}^{*} \otimes \mathfrak{h}$, $\theta_{\mathfrak{m}} \in \wedge^{2} \mathfrak{m}^{*} \otimes \mathfrak{m}, h \in \mathfrak{h}$ and $u_{1}, u_{2} \in \mathfrak{m}$ define $\delta \varphi \in \mathfrak{h}^{*} \otimes \Lambda^{2} \mathfrak{m}^{*} \otimes \mathfrak{m}, Q \varphi \in \mathfrak{h}^{*} \otimes \Lambda^{2} \mathfrak{m}^{*} \otimes \mathfrak{h}$ by

$$
\begin{aligned}
& \delta \varphi(h)\left(u_{1}, u_{2}\right)=\varphi\left(h, u_{1}\right) \cdot u_{2}-\varphi\left(h, u_{2}\right) \cdot u_{1}, \\
& Q \varphi(h)\left(u_{1}, u_{2}\right)=\varphi\left(\varphi\left(h, u_{1}\right), u_{2}\right)-\varphi\left(\varphi\left(h, u_{2}\right), u_{1}\right)-\varphi\left(h, \theta_{\mathfrak{m}}\left(u_{1}, u_{2}\right)\right) .
\end{aligned}
$$

For $v \in\left(\Lambda^{2} \mathfrak{m}^{*} \otimes \mathfrak{m}\right)^{\mathfrak{h}}$ let us also define $p_{v} \in \mathfrak{h}^{*} \otimes \Lambda^{2} \mathfrak{m}^{*} \otimes \mathfrak{h}$ by the formula $p_{v}(h)\left(u_{1}, u_{2}\right)=$ $\varphi\left(h, v\left(u_{1}, u_{2}\right)\right)$ and denote $\Pi_{\varphi}=\left\{p_{\nu} \bmod B^{1}\left(\mathfrak{h}, \Lambda^{2} \mathfrak{m}^{*} \otimes \mathfrak{h}\right)\right\} \subset H^{1}\left(\mathfrak{h}, \Lambda^{2} \mathfrak{m}^{*} \otimes \mathfrak{h}\right)$.

Theorem 1 [3] The Jacobi identity $\operatorname{Jac}\left(v_{1}, v_{2}, v_{3}\right)=0$ with 1 argument from $\mathfrak{h}$ and the others from $\mathfrak{m}$ constrains the cohomology $[\varphi] \in H^{1}\left(\mathfrak{h}, \mathfrak{m}^{*} \otimes \mathfrak{h}\right)$ so:

(1) $[\delta \varphi]=0 \in H^{1}\left(\mathfrak{h}, \Lambda^{2} \mathfrak{m}^{*} \otimes \mathfrak{m}\right)$, whence $\delta \varphi=d \theta_{\mathfrak{m}}$;

(2) $[Q \varphi] \equiv 0 \in H^{1}\left(\mathfrak{h}, \Lambda^{2} \mathfrak{m}^{*} \otimes \mathfrak{h}\right) \bmod \Pi_{\varphi}$, so $Q \varphi=d \theta_{\mathfrak{h}}$ for some choices of $\varphi, \theta_{\mathfrak{m}}$.

Now we are ready to proceed with (a sketch of) the proof of Theorem $3^{+}$. Details of the MAPLE (Differential Geometry) computations can be found in arXiv:1612.09493. 
Proof Let us begin with $\mathfrak{h}=\mathfrak{r}$, when this sub-algebra contains a grading element $s \in \mathfrak{p} \subset$ $\mathfrak{s u}(1,2)$. The cohomology $H^{1}\left(\mathfrak{h}, \mathfrak{m}^{*} \otimes \mathfrak{h}\right)$ has dimension 6 , and a two-dimensional subspace in it satisfies the linear constraint (1) from Theorem 1 [3]. Thus we parametrize $\varphi$ by two essential parameters $c_{1}, c_{2}$. One may then easily derive the equations $c_{1}^{2}=c_{2}^{2}=0$ from Eq. (2) by using that we are working over the real numbers. Hence $[\varphi]=0$, and thus $\mathfrak{g}$ admits a reductive complement to $\mathfrak{h}$, a case which was already completed in [2].

Now let $\mathfrak{h}=\mathfrak{l}_{1} \subset \mathfrak{s u}(1,2)$. In this case the cohomology $H^{1}\left(\mathfrak{h}, \mathfrak{m}^{*} \otimes \mathfrak{h}\right)$ has dimension 4. The full cohomology space satisfies Eq. (1). After solving the linear equation $\delta \varphi=d \theta_{\mathfrak{m}}$, the full remaining Jacobi system is simple enough, so that we compute a Groebner basis and obtain all solutions (in this way we get five distinct families of algebras). All the resulting Lie algebras are isomorphic to $\mathfrak{g}$ from the example above, and $\mathfrak{l}_{1}$ has one-dimensional intersection with $V^{4} \subset \mathfrak{g}$. It is then possible to show that all embeddings of the Lie algebra $\mathfrak{l}_{1}$ into $\mathfrak{g}$ with the required intersection, and which admit an invariant almost complex structure on the quotient $\mathfrak{g} / \mathfrak{l}_{1}$, are conjugate in $\mathfrak{g}$. The non-degeneracy of the Nijenhuis tensor can be verified from the structure constants of $\mathfrak{g}$ given in the example.

Finally let $\mathfrak{h}=\mathfrak{l}_{0}$. Then the cohomology $H^{1}\left(\mathfrak{h}, \mathfrak{m}^{*} \otimes \mathfrak{h}\right)$ has dimension 10 . The solution space of (1) has dimension 5. After solving the linear equation $\delta \varphi=d \theta_{\mathfrak{m}}$, the full Jacobi system [and even Eq. (2) alone] is too complex for Groebner basis methods or direct solvers, and a full solution seems to be out of reach. However, we compute the Nijenhuis tensor of the corresponding structure $J$ in terms of the Lie algebra parameters, without solving the Jacobi system. The coefficients of this depend only on 10 parameters (out of the 145 variables of the Jacobi system). We then show that modulo the Jacobi system, the Nijenhuis tensor has rank at most 2 , and hence it is degenerate and we are done.

\section{References}

1. Alekseevsky, D.V., Kruglikov, B.S., Winther, H.: Homogeneous almost complex structures in dimension 6 with semi-simple isotropy. Ann. Glob. Anal. Geom. 46, 361-387 (2014)

2. Kruglikov, B., Winther, H.: Almost complex structures in 6D with non-degenerate Nijenhuis tensors and large symmetry groups. Ann. Glob. Anal. Geom. 50, 297-314 (2016)

3. Kruglikov, B., Winther, H.: Reconstruction from Representations: Jacobi via Cohomology. arXiv:1611.05334 (2016) 